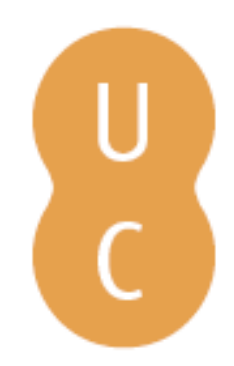

\title{
nombalina
}

\section{O b-learning no desenvolvimento de competências em recuperação da informação}

\author{
Autor(es): $\quad$ Serrano, Anabela \\ Publicado por: Imprensa da Universidade de Coimbra \\ URL \\ persistente: URI:http://hdl.handle.net/10316.2/31912 \\ DOI: $\quad$ DOI:http://dx.doi.org/10.14195/978-989-26-0319-3_26
}

Accessed : $\quad$ 26-Apr-2023 13:13:20

A navegação consulta e descarregamento dos títulos inseridos nas Bibliotecas Digitais UC Digitalis, UC Pombalina e UC Impactum, pressupõem a aceitação plena e sem reservas dos Termos e Condições de Uso destas Bibliotecas Digitais, disponíveis em https://digitalis.uc.pt/pt-pt/termos.

Conforme exposto nos referidos Termos e Condições de Uso, o descarregamento de títulos de acesso restrito requer uma licença válida de autorização devendo o utilizador aceder ao(s) documento(s) a partir de um endereço de IP da instituição detentora da supramencionada licença.

Ao utilizador é apenas permitido o descarregamento para uso pessoal, pelo que o emprego do(s) título(s) descarregado(s) para outro fim, designadamente comercial, carece de autorização do respetivo autor ou editor da obra.

Na medida em que todas as obras da UC Digitalis se encontram protegidas pelo Código do Direito de Autor e Direitos Conexos e demais legislação aplicável, toda a cópia, parcial ou total, deste documento, nos casos em que é legalmente admitida, deverá conter ou fazer-se acompanhar por este aviso.

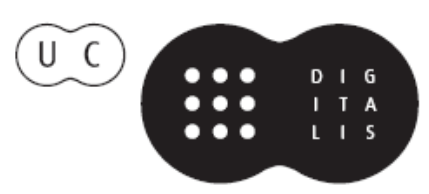


Maria Manuel Borges

Elias Sanz Casado

Coordenação

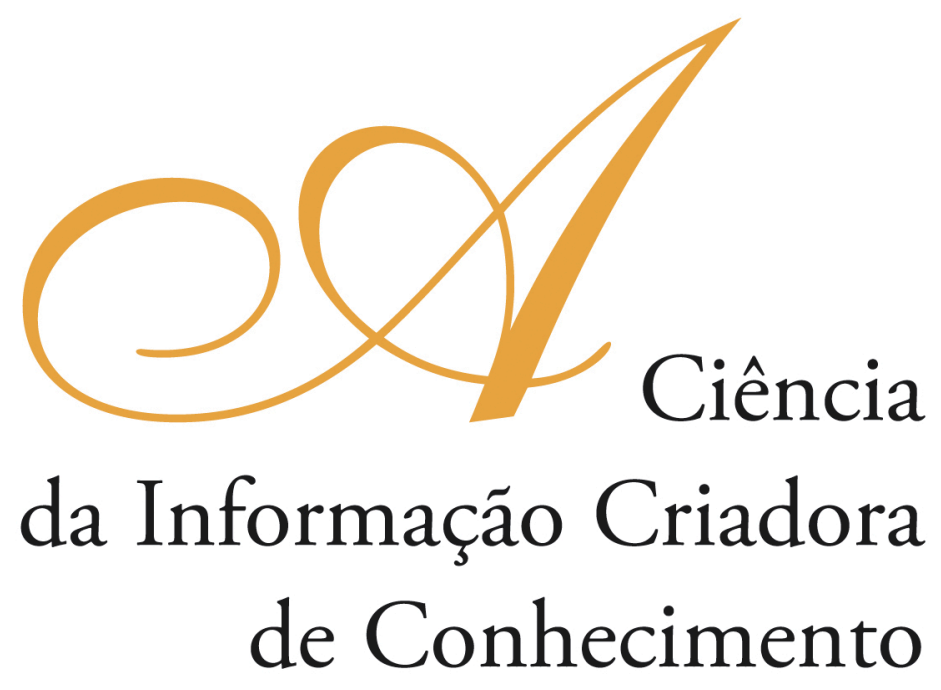

Vol. I

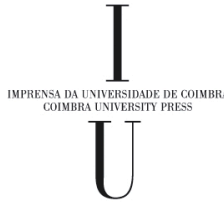

- COIMBRA 2009 


\title{
O B-LEARNING NO DESENVOLVIMENTO DE COMPETÊNCIAS em Recuperação da Informação
}

\author{
Anabela Serrano \\ Instituto Politécnico do Porto (Portugal)
}

\section{Resumo}

A adequação dos cursos do ensino superior ao processo de Bolonha implicou, em muitos casos, uma mudança na selecção das várias abordagens possíveis, consoante a área científicotécnica e a essência de cada unidade curricular (UC), ao processo de ensino/aprendizagem. A tónica deixou de estar na transmissão de conhecimentos e passou para o desenvolvimento de competências mediante uma participação activa do aluno no processo em si. Em ciência da informação $(\mathrm{CI})$, a área disciplinar de recuperação da informação (RI) constitui um desafio aliciante na aplicação dos princípios de Bolonha quer por pertencer ao "core" de CI quer pelo facto de ser uma área onde sempre houve uma enorme actividade de investigação. Através da análise da literatura mais recente acerca do ensino e da aprendizagem de RI, apresenta-se um caso prático de desenvolvimento de uma UC em que se utiliza o b-learning como estratégia de desenvolvimento de competências em futuros profissionais de informação.

Para além das competências a desenvolver nos discentes, da selecção do programa da UC e dos métodos/técnicas de ensino, são ainda explorados e discutidos os métodos de avaliação que possam ser mais adequados numa UC com estas características.

Mediante a análise de dados relativos à avaliaçáo do desempenho dos discentes pelo docente e à avaliaçáo da UC pelos discentes, conclui-se que a adopçấo do sistema de ensino misto blearning tem um impacto positivo no desenvolvimento de competências de RI. Por último, pondera-se sobre novos desenvolvimentos e experiências no âmbito da UC "Recuperação da Informação" do curso de Ciências e Tecnologias da Documentação e Informação da Escola Superior de Estudos Industriais e de Gestão do Instituto Politécnico do Porto.

\begin{abstract}
The adequacy of higher education degrees to the Bologna process implied, in many cases, a change in the selection of the various possible approaches, depending on the scientific-technical domain and on the essence of each curricular unit (CU), to the teaching/learning process. The accent switched from knowledge transmission to skills development through an active participation of the student inside the process itself. In Information Science (IS), the subject area of Information Retrieval (IR) is an exciting challenge in applying the principles of Bologna either by belonging to the "core» of IS either by being an area where there has always been a great research activity. Through a review of the latest literature about teaching and learning in IR, a case study is presented on developing a CU where b-learning is used as a strategy for developing skills in future information professionals.

Beyond the skills to develop in students, the selection of the CU program and the methods / techniques of teaching, are also explored and discussed the evaluation methods that may be more appropriate in a $\mathrm{CU}$ with these characteristics. Through the analysis of data concerning the performance evaluation of students by the teacher and the evaluation of the $\mathrm{CU}$ by the students, it is concluded that the adoption of the mixed education system b-learning has a
\end{abstract}


positive impact on the development of skills of IR. Finally, new developments and experiences are considered within the CU «Information Retrieval» in the degree of Science and Technologies of Documentation and Information of the Escola Superior de Estudos Industriais e de Gestão at the Instituto Politécnico do Porto.

\section{Introdução}

Esta comunicação consiste numa reflexão acerca do desenvolvimento curricular da disciplina de "Recuperação da Informação", no âmbito da adequação ao processo de Bolonha do curso de licenciatura em Ciências e Tecnologias da Documentação e Informação da Escola Superior de Estudos Industriais e de Gestão do Instituto Politécnico do Porto (ESEIG/IPP), e da adopção do sistema de ensino misto b-learning (ou blended learning), em detrimento de outras opçóes, com o objectivo de promover, da melhor forma, o desenvolvimento de competências necessárias ao profissional de informação.

Das inúmeras pesquisas realizadas em vários recursos de informação, para o período de 2004-2009, foram considerados relevantes para o trabalho aqui apresentado, de entre os documentos recuperados, doze (12) documentos publicados entre 2005 e 2009 e que constam das referências bibliográficas apresentadas no final deste documento.

O corpo deste documento estrutura-se da seguinte forma:

- Adequação a Bolonha

- Desenvolvimento curricular

- Sistema de ensino

- b-learning - ensino, comunicação e interacção

- Competências profissionais em contexto educacional

\section{Adequação a Bolonha}

A assinatura da "Declaração de Sorbonne» pelos ministros da educação de quatro países da União Europeia (França, Itália, Reino Unido e Alemanha) a 25 de Maio de 1998, conduziu, um ano depois, à assinatura da "Declaração de Bolonha" pelos ministros da educação de 29 países europeus - a 19 de Junho de 1999 -, a qual despoletou um processo de reestruturação e harmonizaçáo do ensino superior no espaço europeu que ficou conhecido pela designação de "O Processo de Bolonha". Era objectivo dos subscritores desta declaração, nos quais Portugal se inclui, criar uma Europa competitiva e líder da sociedade da informação em que vivemos baseada na excelência ao mais alto nível do conhecimento. ${ }^{1}$

Dez anos volvidos, o processo está quase concluído, tendo o ensino superior português sofrido uma reforma alargada que, entre outros aspectos, obrigou as instituiçóes a adequarem a sua oferta formativa às novas exigências legislativas e estratégicas.

\footnotetext{
${ }^{1}$ A avaliação de serviços de informação do ensino superior (Serrano, 2003), passim.
} 
Assim, em 2005, foi alterada a lei de bases do sistema educativo ${ }^{2}$ português definindo-se:

- «A criação de condições para que todos os cidadãos possam ter acesso à aprendizagem ao longo da vida, modificando as condiçóes de acesso ao ensino superior para os que nele não ingressaram na idade de referência, atribuindo aos estabelecimentos de ensino superior a responsabilidade pela sua selecção e criando condiçóes para o reconhecimento da experiência profissional;

- A adopçáo do modelo de organização do ensino superior em três ciclos;

- A transição de um sistema de ensino baseado na ideia de transmissão de conhecimentos para um sistema baseado no desenvolvimento de competências;

- A adopçáo do sistema europeu de créditos curriculares (ECTS - European Credit Transfer and Accumulation System), baseado no trabalho dos estudantes.» ${ }^{3}$

Em Março de 2006, foi publicado o diploma legal ${ }^{4}$ que se refere aos graus e diplomas e que estabelece as formas de adequação dos cursos existentes no ensino superior de modo a organizar este sistema de ensino em três (3) ciclos tal como consta no diploma atrás referido. $\mathrm{Na}$ sequência da sua entrada em vigor, as instituiçóes de ensino superior tiveram de levar a cabo as medidas aí estabelecidas nos prazos fixados. $\mathrm{Na}$ Escola Superior de Estudos Industriais e de Gestão, o curso de licenciatura bietápica em Ciências e Tecnologias da Documentação e Informação (CTDI), com a duração de cinco anos, foi adequado a um curso de licenciatura com a mesma designaçáo ${ }^{5}$ e a duração de três anos. Criar um grau de ensino, com pouco mais de metade da duração do existente, assegurando as competências indispensáveis ao profissional de informação, e sem eliminar o estágio profissional, tornou-se uma tarefa complexa, dificultada, ainda mais, pela exiguidade do prazo conferido pelo Ministério da Ciência, Tecnologia e do Ensino Superior (MCTES).

A criação da unidade curricular (UC) "Recuperação da Informação" (RI) no curso de CTDI, sucessora da UC de OPISM ${ }^{6}$ do plano de estudos anterior à adequação, foi algo controversa tendo sido alvo de contestação por parte de alguns colegas do grupo de trabalho. Não obstante, a sua importância inequívoca no âmbito da Ciência da Informação, constituindo uma das áreas disciplinares do seu "core", fez com que o grupo de trabalho optasse pela sua criação.

\section{Desenvolvimento curricular}

Tendo por base duas importantes obras, ambas publicadas em 2005, como o European Curriculum Reflections on Library and Information Science, e o EuroReferencial

${ }^{2}$ Lei no 49/2005 de 30 de Agosto.

3 Decreto-Lei no 74/2006 de 24 de Março, DR No 60, Ia Série-A, p. 2242.

${ }^{4}$ Idem, p. 2242-2257.

5 O grupo de trabalho de CTDI propôs a alteração da designação do curso para "Ciência e Tecnologias da Informação", mas a mesma não foi aceite pela tutela.

${ }^{6}$ Organização e Pesquisa Interactivas em Suportes Múltiplos. 
$I-D$, e múltiplas pesquisas em vários recursos de informação disponíveis na Internet, levou-se a cabo um estudo comparativo a nível europeu e a nível nacional de cursos análogos.

Relativamente à unidade curricular de RI, o capítulo 4 da obra publicada pela The Royal School of Library and Information Science, na Dinamarca, sob o patrocínio da União Europeia e com contributos de cerca de 150 especialistas em Ciência da Informação geridos por 12 grupos de trabalho aos quais pertenciam 40 a 50 especialistas, foi uma referência importante e bastante elucidativa. David Bawden geriu um grupo de trabalho constituído por mais cinco especialistas provenientes de vários países da União Europeia, sendo Portugal representado por Inês Cordeiro, cujas reflexôes se consubstanciaram em cerca de 17 páginas sob o título "Information Seeking and Information Retrieval" (IS\&R). Segundo os autores, esta é uma temática demasiado vasta para que se possa pretender definir um modelo curricular único. Identificam três aspectos principais relacionados entre si: o comportamento informacional (objecto da criação de uma UC independente em CTDI), a pesquisa de informação e a recuperação de informação, e consideram que, indubitavelmente, a IS\&R faz parte da essência ("core") da CI. Afirmam, ainda, que em conjugação com a Organização do Conhecimento (ou análise e representação da informação) - embora a escolha de, apenas, esta parceria possa ser questionável - constitui a verdadeira essência da Ciência da Informação.

Ainda de acordo com os autores do capítulo acima referido, esta área temática deverá ter lugar cativo em qualquer curso de Ciência da Informação:

«It should be included in any LIS course, at any level. It has relations with several other themes within the discipline, particularly with $\mathrm{KO}$ and with Information Literacy and Learning. It is clearly an important 'European theme', and a crucial part of any European LIS curriculum, though there are no uniquely 'European values' associated with it. (...)

(...)There are numerous concepts, models and frameworks for the whole IS\&R area, and these may be used as the basis for the organisation of a curriculum. The core of the analysis of this chapter is the presentation and discussion of set of 28 topics, covering the whole theme, from which courses at a variety of levels (Bachelor, Master, or professional development), depth, and subject focus may be constructed.» ${ }^{7}$

$\mathrm{Na}$ conclusão do capítulo, referem que a IS\&R pode ser considerada a essência da CI:

«Together with KO and ILL, IS\&R makes up one of the cores - indeed, it may be argued, the core - of the LIS discipline. Any effective modern LIS curriculum must include an appropriate treatment of this theme.. ${ }^{8}$

\footnotetext{
${ }^{7}$ European Curriculum Reflections on Library and Information Science (Bawden, 2005), p. 84-85.

${ }^{8}$ Idem, p. 98.
} 
Vilar (2009) apresenta estes 28 tópicos numa tabela9 ${ }^{9}$ o que facilita a sua análise.

Em 2006, a consulta desse tópicos, bem como de literatura de referência em RI, permitiu-nos seleccionar aquele ou aqueles que melhor se adequam ao perfil da nossa unidade curricular, contextualizando-a no novo curso a cujo desenvolvimento curricular se tinha dado início. Tendo optado por criar uma UC para o comportamento informacional, faltava-nos cobrir os outros 2 aspectos no currículo do novo curso: a pesquisa da informação e a recuperação da informação. Sendo passíveis de distinção, acabam por ser duas faces da mesma moeda, duas facetas do acesso à informação, pelo que a designação adoptada, que deveria ser o mais curta possível de acordo com linhas de orientaçáo do MCTES, acabou por ser "recuperação da informação". Para além do mais, a recuperação da informação agrega mais tópicos e a maioria do conteúdo considerado de interesse para um curso cuja vertente tecnológica tem maior peso do que a vertente social e humana, podendo esta ser explorada na UC "Comportamento Informacional”.

As competências a adquirir pelos alunos foram identificadas com base no EuroReferencial $I-D^{10}$ e na experiência profissional adquirida em contexto organizacional ao longo de década e meia de presença no mercado de trabalho:

\section{Competências gerais:}

1. Compreender e definir conceitos básicos de recuperação de informação;

2. Desenvolver a capacidade de análise, e a de avaliação e diagnóstico;

3. Aplicar os conhecimentos adquiridos a novas situaçóes equacionando a resolução de problemas no domínio profissional;

4. Implementar projectos.

\section{Competências específicas:}

1. Analisar pedidos de informação complexos, elaborar estratégias de pesquisa e reproduzir o resultado da pesquisa;

2. Identificar e seleccionar fontes de informação;

3. Dominar instrumentos e técnicas de pesquisa;

4. Diagnosticar e avaliar sistemas de recuperação de informação (SRI)

De forma a permitir a aquisição destas competências pelos alunos, foi criado o currículo que consta no plano de estudos aprovado em 2006, e que prevê o seguinte programa:

- Introdução à Recuperação da Informação (RI) - o processo de RI

- Sistemas de RI

- Modelos de RI

- Processamento de interrogaçóes

- Recuperação de Informação na Web

- A interface do utilizador e a visualização

- Avaliaçáo de Sistemas de RI

\footnotetext{
9 V. Anexo 1.

${ }^{10}$ Op. Cit., p. 45-46.
} 
Estes tópicos são passíveis de vários desenvolvimentos, e de serem dados com maior ou menor profundidade em função do contexto de ensino em que se inserem. Na base da selecçáo dos mesmos, estiveram os seguintes objectivos educacionais:

- Conhecimento dos fundamentos em RI;

- Formação em estratégias de pesquisa;

- Conhecimento de sistemas de recuperação da informação (SRI).

Estes mesmos objectivos foram identificados para RI por Fernández-Luna (2009) na área de Ciências dos Computadores (CC), apesar daquela não fazer parte da essência de CC.

Os métodos de ensino adoptados são múltiplos, e são (re)combinados em funçâo do perfil de cada turma, privilegiando-se o método activo em detrimento do expositivo.

O método de avaliação seleccionado constitui um misto de avaliação contínua (trabalhos práticos) e de avaliação final (exame com parte teórica e parte prática) por se considerar importante a existência de provas em que seja possível analisar diferentes aptidóes dos alunos. A parte prática do exame pretende reproduzir em sala de aula exercícios de aplicação prática passíveis de ocorrerem em contexto profissional, à semelhança daquilo que sucede, ao longo do semestre, nas aulas da UC aquando da resolução de exercícios práticos.

\section{Sistema de ensino}

Convencional, e-learning ou b-learning?

Dadas as características da RI, e dado o facto de existir uma significativa percentagem de alunos trabalhadores-estudantes no curso de CTDI, a escolha recairia sempre no b-learning. Adicionalmente, ainda não é plausível assegurar, em função de diversos factores que passam por níveis de decisão e de alocação de recursos variados, um curso, integralmente, em sistema de ensino à distância ou e-learning na ESEIG/IPP. Deste modo, facultar uma UC, isoladamente, em e-learning não traria vantagens acrescidas de per si. Um sistema de blended learning ou b-learning, implica a disponibilizaçáo de conteúdos numa plataforma de e-learning a par da realização de aulas em ambiente de sala de aula física, e, em virtude do processo de Bolonha, a par da disponibilizaçáo de apoio tutorial em ambiente de sala de aula (para além da carga horária lectiva) e do atendimento no gabinete.

Disponibilizar conteúdos online implica a análise de uma série de aspectos que deverão ser tidos em conta: tipo de conteúdo de acordo com a sua actualização e de acordo com a sua tipologia. Conteúdos, maioritariamente, estáveis como os conceitos fundamentais de RI não necessitam de grande actualização e podem ser facultados em formatos mais estáticos, enquanto que conteúdos não estabilizados como tópicos objecto de investigação deverão ser facultados em formatos mais dinâmicos (Henrich, 2009).

A unidade curricular do RI é disponibilizada online na plataforma de e-learning adoptada na ESEIG/IPP: o Moodle. Esta plataforma apresenta algumas limitaçóes que depressa são esquecidas quando nos recordamos do facto de que a mesma não acarreta qualquer encargo financeiro para a instituição. Há várias plataformas comercializadas 
extremamente eficazes, mas as restriçôes orçamentais são determinantes na selecção deste tipo de soluçóes. Os conteúdos estão dispostos por tópicos e agrupam os conceitos fundamentais, os conteúdos objecto de investigaçáo (incidindo nos 3 últimos pontos do programa), os exercícios práticos e os trabalhos práticos, os SRI, os motores de pesquisa, etc. Os formatos disponibilizados são: pdf, html, xml, ppt, doc; e, também, ligaçôes para canais do Youtube (vídeos da University of North Carolina at Chapel Hill - Gary Marchionini; do Google), entre outros ${ }^{11}$. Ficheiros de vídeo não são carregados, directamente, devido às limitaçóes de upload da plataforma - mesmo assim, e a título de exemplo, refira-se que no ano lectivo de 2008/2009 não foi possível importar a disciplina do ano lectivo anterior pelo facto de o ficheiro ser muito maior (cerca de $162 \mathrm{MB})$ do que os $64 \mathrm{MB}$ permitidos...

\section{b-learning - ensino, comunicação e interacção}

Desenvolver competências implica um papel activo por parte do aluno e a promoção da interactividade por parte do docente. Comunicar deve conduzir à interacção, já que, tal como demonstrou Shannon, no processo info-comunicacional o canal de comunicaçáo existente entre emissor e receptor permite o feedback.

Pesquisar/recuperar informação implica, acima de tudo raciocínio e espírito crítico.

Para ser bem sucedido na resolução de um exercício (o que exige a utilização simultânea de quase todas as competências enunciadas para a UC), um aluno deve:

1. conseguir analisar e representar o conteúdo de uma necessidade de informação,

2. conhecer inúmeros recursos de informação, ou saber como aceder-lhes, e saber avaliar quais os recursos mais adequados a cada caso,

3. dominar as técnicas/funcionalidades de pesquisa passíveis de serem utilizadas num SRI,

4. saber elaborar uma síntaxe de pesquisa ou query criando alternativas sucessivas com rapidez,

5. saber interpretar os resultados do SRI e equacionar nova estratégia de pesquisa se necessário.

Boas bases conceptuais em RI, aptidão tecnológica, capacidade de abstracção, velocidade de processamento cognitivo e espírito crítico/capacidade de avaliação são, portanto, requisitos que facilitam o bom desempenho académico e profissional nesta área central e essencial da Ciência da Informação.

Aulas convencionais em que são projectados slides, acetatos, ou outro tipo de documentos (náo raras vezes, apenas, lidos) sem que o debate seja suscitado ou estimulado por parte do docente, ou aulas ditas "bolonhesas" em que o docente permanece na sala (quando náo está no gabinete) sem interagir com os alunos nem

11 Não constitui caso único, apesar de a ideia ter ocorrido sem outras influências prévias - outros docentes de RI de outros países têm a mesma prática de forma a poderem cobrir um espectro maior da temática de RI (Jones, 2007). 
sequer assumindo o papel de tutor no esclarecimento de dúvidas e na orientação dos trabalhos práticos cujos enunciados elaborou, não promovem o desenvolvimento de competências nem estimulam o raciocínio. ${ }^{12}$

Debates, projecção de vídeos com pausas para discussão e anotação de tópicos no quadro ou num acetato ou slide, técnicas de "brainstorming", entre outras possibilidades, são técnicas de ensino que podem promover a interactividade em ambiente de sala de aula.

Já nas plataformas digitais, a comunicação pode ser síncrona, tal como em ambiente de sala de aula (física ou virtual), ou assíncrona. Como exemplo de comunicação síncrona temos o Messenger, o ICQ, o Google, o Skype, o Second Life (que permite ambiente de sala de aula ou de contexto profissional virtual), etc. Como exemplo de comunicação assíncrona temos o email, os fóruns, os blogues, os sites com comentários e co-edição (Google Sites), etc.

\section{Competências profissionais em contexto educacional}

Neste novo contexto educacional europeu, torna-se premente a criação de estímulos e a colocação de desafios aos alunos de forma a conseguir a adesão, não só dos alunos ditos "profundos", dos alunos ditos "superficiais" (Jones, 2007).

A criação de estímulos já ficou exemplificada acima.

A colocação de desafios pode traduzir-se na criação de competiçôes que atribuam prémios aos vencedores. Na unidade curricular de RI em análise, foram criadas as “Olimpíadas de RI", no ano lectivo de 2006/2007 (1 $1^{\text {a }}$ ocorrência da UC no 2o semestre), que consistem numa competição em que os alunos participantes resolvem uma série de exercícios práticos (nos moldes do enunciado da parte prática do exame), mas com cronómetro. Os vencedores (os 3 mais rápidos de entre aqueles que obtiverem a melhor classificação) obtêm um crédito de pontos variável de acordo com a posição obtida, previamente anunciado, na classificação de uma prova de avaliação da UC (geralmente, na parte prática do exame). A classificação final pode ser beneficiada até um máximo de $10 \%{ }^{13}$. Na generalidade, os alunos aderem com entusiasmo e consideram que a existência do cronómetro faz com que tenham melhores resultados na parte prática do exame final, aumentando os níveis de confiança e diminuindo o stress durante a prova de avaliação.

As aulas tutoriais previstas nos horários do curso têm sido mais valorizadas pelos docentes do que pelos alunos, possivelmente, pelo facto de não estarem sujeitas a regime presencial. Todavia, se o estilo tutorial fôr adoptado pelos docentes ao longo do semestre, em todos os contactos com os alunos, nota-se uma melhor adesão dos alunos no cumprimento das tarefas agendadas.

\footnotetext{
12 Teaching information retrieval using research questions to encourage creativity and assess understanding (Jones, 2007), passim.

13 Outros autores mencionam experiências semelhantes em artigo publicado este ano (Henrich, 2009).
} 
Os enunciados dos exercícios e dos trabalhos práticos, bem como da parte prática do exame (ponderação de $40 \%$ para trabalhos $+30 \%$ para a prática do exame $=70 \%$ na classificação final) procuram recriar situaçóes reais passíveis de ocorrerem em contexto profissional.

\section{Conclusão}

O sistema de ensino adoptado tem-se revelado adequado ao desenvolvimento de competências em Recuperação da Informação, pelo que o b-learning continuará a ser a nossa opção nos próximos três anos lectivos.

As taxas de aprovação têm sido positivas ao longo destes 3 últimos anos lectivos (2006/2007 a 2008/2009), bem como a avaliaçáo da UC pelos alunos. Os estágios profissionais em que as competências adquiridas em RI foram colocadas à prova ou constituíram o tema central dos mesmos deixaram uma impressão positiva nas instituiçóes acolhedoras de estágio e/ou nos estagiários.

Um inquérito aos alunos acerca das especificidades da UC será uma forma de poder aprofundar este trabalho e de pugnar pela melhoria contínua do processo de ensino/aprendizagem no seio da unidade curricular, indicando-nos novos caminhos ou inflexôes sempre que necessário. Pretende-se introduzir este meio de controlo de qualidade, em breve, no âmbito da UC.

Nos próximos anos lectivos, seria útil poder utilizar outras funcionalidades no sistema de e-learning da ESEIG, tais como o webcast ou o podcast.

Ter-se-á atenção à publicação de novos manuais de conceitos básicos e fundamentais em RI, bem como aos congressos da temática como o TREC, o SIGIR, o TLIR, etc.

Continuar-se-á a acompanhar a investigação em RI e a participar em redes de investigação que permitam a partilha de materiais pedagógicos (indo para além da utilização de plataformas colaborativas disponíveis online como o YouTube ou o Slide Share), possibilitando aos alunos o acesso ao que de melhor se faz em RI, no resto do mundo, em universidades de excelência.

Tem-se, igualmente, por objectivo continuar a actualizar os conteúdos da UC e a apostar na interactividade em ambiente de sala de aula, e quem sabe se, num futuro próximo, em webcast ou no Second Life...

\section{Referências Bibliográficas}

BAEZA-YATES, R. \& RIBEIRO-NETO, B. (1999). Modern information retrieval. New York, USA: ACM Press.

BATES, J., BAWDEN, D., CORDEIRO, I., STEINEROVÁ, J., VAKKARI, P. \& VILAR, P. (2005). Information seeking and research. In: Kajberg, L. \& Lørring, L. (Eds.) European Curriculum Reflections on Library and Information Science Education, (pp. 84-100). Copenhagen, Denmark: The Royal School of Library and Information Science.

BAWDEN, D., BATES, J., STEINEROVU, J., VAKKARI, P. \& VILAR, P. (2007). Information retrieval curricula: contexts and perspectives. In A. McFarlane, J. M. Fernandez Luna, I. 
Ounis, \& J. F. Huete (Eds.), Proceedings of the First International Conference on Teaching and Learning Information Retrieval, London, UK.

BORGMAN, C. L. (2006). What can studies of e-learning teach us about collaboration in eresearch? Some findings from digital libraries studies. Computer Supported Cooperative Work, 15:4, 359-383. DOI 10.1007/s10606-006-9024-1

EUROPEAN COUNCIL OF INFORMATION ASSOCIATIONS (2005). Euro-referencial I-D. Lisboa, Portugal: INCITE.

FERNÁNDEZ-LUNA, J. M., HUETE, J. F., MacFARLANE, A. \& EFTHIMIADIS, E. N. (2009). Teaching and learning in information retrieval. Information Retrieval, 12:2, 201 226. DOI $10.1007 / \mathrm{s} 10791-009-9089-9$

HENRICH, A. \& SIEBER, S. (2009). Blended learning and pure e-learning concepts for information retrieval: experiences and future directions. Information Retrieval, 12:2, 117-147. DOI 10.1007/s10791-008-9079-3

JONES, G. J. F. (2007). Teaching information retrieval using research questions to encourage creativity and assess understanding. In A. McFarlane, J. M. Fernandez Luna, I. Ounis, \& J. F. Huete (Eds.), Proceedings of the First International Conference on Teaching and Learning Information Retrieval, London, UK.

HALTTUNEN, K., JARVELIN, K. (2005). Assessing learning outcomes in two information retrieval learning environments. Information Processing and Management, 41:4, 949-972.

LEE, S., FOX, E. A., MARCHIONINI, G., VELACSO, J., ANTUNES, G. \& BORBINHA, J. (2009). Virtual DL poster sessions in Second Life. JCDL'09, June 15-19, 2009, Austin, Texas, USA. ACM 978-1-60558-322-8/09/06.

McMARTIN, F., IVERSON, E., WOLF, A., MORRILL, J., MORGAN, G. \& MANDUCA, C. (2008). The use of online digital resources and educational digital libraries in higher education. International Journal on Digital Libraries, 9:1, 65-79. DOI 10.1007/s00799008-0036-y

MIZZARO, S. (2007). Teaching of web information retrieval: web first or IR first? In A. McFarlane, J. M. Fernandez Luna, I. Ounis, \& J. F. Huete (Eds.), Proceedings of the First International Conference on Teaching and Learning Information Retrieval, London, UK.

SERRANO, A. (2003). A avaliação de serviços de informação do ensino superior. Páginas $\boldsymbol{a} \mathfrak{b} \boldsymbol{b}$ : arquivos e bibliotecas, 12, 7-65.

VILAR, P. \& ZUMER, M. (2009). The Bologna Reform at the department of library and information science and book studies, University of Ljubljana. Information Retrieval, 12:2, 102-116. DOI 10.1007/s10791-008-9077-5 


\section{Anexo 1}

Tópicos IS\&R (Bates, 2005) apresentados por Vilar (2009) ${ }^{14}$ :

\begin{tabular}{|c|c|}
\hline 1 & Basic concepts and relationships \\
\hline \multirow[t]{4}{*}{ Gen } & Relationships between HIB, IS, IR \\
\hline & Three perspectives: human/user, culture, system \\
\hline & Concepts: information and knowledge; documents; typology of information resources \\
\hline & Relevant research methodologies; laboratory, operational, qualitative, quantitative \\
\hline 2 & Overview of HIB \\
\hline \multirow[t]{4}{*}{ HIB } & Frameworks, concepts, models, theories \\
\hline & Research approaches and methods \\
\hline & Example topics \\
\hline & Historical development of studies \\
\hline 3 & Overview of IS \\
\hline \multirow[t]{4}{*}{ IS } & Frameworks, concepts, models, theories \\
\hline & Research approaches and methods \\
\hline & Example topics \\
\hline & Historical development of studies \\
\hline 4 & Overview of IR \\
\hline \multirow[t]{5}{*}{ IR } & Frameworks, concepts, models, theories \\
\hline & Components of retrieval systems \\
\hline & Research approaches and methods \\
\hline & Example topics \\
\hline & Historical development of studies \\
\hline 5 & Human information behaviour: people \\
\hline \multirow[t]{3}{*}{ HIB } & Individuals and groups \\
\hline & Occupation, age, activity, etc. \\
\hline & Characteristics: cognitive, social, cultural, organisational \\
\hline 6 & Human information behaviour: sources and places \\
\hline \multirow[t]{3}{*}{ HIB } & Channels and media \\
\hline & Print, electronic, formal, informal, mass, local, ICTs \\
\hline & Places \& spaces - libraries, information centres, archives, museums, information grounds \\
\hline 7 & Human information behaviour: patterns of behaviour \\
\hline \multirow[t]{2}{*}{ HIB } & Browsing, encountering, avoidance, anxiety, advantages of lack of information, overload \\
\hline & Innovation and creativity \\
\hline 8 & Information needs; nature, typology \\
\hline HIB & Identifying information needs; users and non-users \\
\hline 9 & Information literacy \\
\hline \multirow[t]{2}{*}{ HIB } & Place of seeking/retrieval in wider context \\
\hline & Teaching and supporting users to retrieve \\
\hline 10 & Organising and using information \\
\hline \multicolumn{2}{|r|}{ 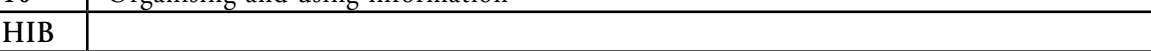 } \\
\hline 11 & Role of information professionals \\
\hline
\end{tabular}

${ }^{14}$ Disponível em http://informationr.net/ir/12-4/colis/colise03.html 


\begin{tabular}{|c|c|}
\hline HIB & \\
\hline 12 & Information seeking in context \\
\hline IS & Occupational, professional, everyday life, etc. \\
\hline 13 & Information seeking in specific domains (subjects) \\
\hline \multirow[t]{2}{*}{ IS } & Relation to domain analysis \\
\hline & Domain specific resources \\
\hline 14 & Strategies and tactics for information seeking \\
\hline IS & Task-based and cognitive etc. \\
\hline 15 & Relevance and satisfaction \\
\hline IS & Concepts, typology, history, empirical studies \\
\hline 16 & Person-centred information services \\
\hline IS & Developing services around needs, using research findings \\
\hline 17 & Historical development of IR systems \\
\hline IR & Associated IT: retrieval in different media - print, digital, network \\
\hline 18 & Retrieval interfaces \\
\hline \multirow[t]{2}{*}{ IR } & HCI, usability testing, personalisation \\
\hline & Machine interfaces and interoperability, visualisation \\
\hline 19 & Typology of retrieval systems \\
\hline \multirow[t]{5}{*}{ IR } & DBMS, factual/numeric systems \\
\hline & Bibliographic databases, full-text retrieval, e-journals, content management systems \\
\hline & OPACs, digital library, managing digital resources \\
\hline & Internet search engines, subject gateways, 'hidden web', semantic web \\
\hline & Enterprise and knowledge management systems (Autonomy, Verity, Google, etc.) \\
\hline 20 & Specialised retrieval \\
\hline IR & $\begin{array}{l}\text { E.g. multimedia, images, audio, sounds, music, fiction, chemical structure, genome and } \\
\text { protein sequence }\end{array}$ \\
\hline 21 & Intelligent systems and techniques; cognitive aspects \\
\hline \multirow[t]{4}{*}{ IR } & Intelligent agents, $\mathrm{AI}$ \\
\hline & Data / text mining \\
\hline & Question-answering systems, recommender systems \\
\hline & Cyc \\
\hline 22 & Retrieval tactics \\
\hline IR & General and specific \\
\hline 23 & Citation searching, bibliometrics, webliometrics \\
\hline \multicolumn{2}{|l|}{ IR } \\
\hline 24 & Retrieval language \\
\hline \multirow[t]{2}{*}{ IR } & Natural language processing, automatic indexing, classification, summarisation \\
\hline & Multilingual systems, CLIR \\
\hline 25 & Metadata and controlled vocabularies \\
\hline \multirow[t]{5}{*}{ IR } & Controlled vocabularies in retrieval \\
\hline & $\begin{array}{l}\text { Ontologies, subject headings, thesauri, taxonomies, classification, RDF, topic maps, } \\
\text { concept retrieval / topic retrieval / latent semantic retrieval }\end{array}$ \\
\hline & Metadata and retrieval \\
\hline & Intellectual metadata creation: cataloguing, indexing, abstracting \\
\hline & Format and content standards \\
\hline 26 & Evaluation of systems and services \\
\hline IR & IR system evaluation: TREC, metrics and other performance measures \\
\hline
\end{tabular}




\begin{tabular}{|l|l|}
\hline & User-oriented evaluation of information seeking and searching \\
\hline 27 & System design based on research findings \\
\hline IR & \\
\hline 28 & New developments and future trends in HIB, IS and IR \\
\hline Gen & Current research topics \\
\hline & Evaluating research, evidence-based practice \\
\hline
\end{tabular}

